\title{
On the Use of Tunable Power Splitter for Simultaneous Wireless Information and Power Transfer Receivers
}

\author{
Abdul Quddious $\mathbb{D},{ }^{1}$ Muhammad Ali Babar Abbasi $\mathbb{D},{ }^{2}$ Muhammad Haroon Tariq $\mathbb{D},{ }^{1}$ \\ Marco A. Antoniades $\left(\mathbb{D},{ }^{3}\right.$ Photos Vryonides ${ }^{D},{ }^{1}$ and Symeon Nikolaou ${ }^{1}{ }^{1}$ \\ ${ }^{1}$ Department of Electrical Engineering, Frederick University, Nicosia, Cyprus \\ ${ }^{2}$ Centre for Wireless Innovation (CWI), The Institute of Electronics, Communications and Information Technology (ECIT), \\ Queen's University Belfast, Belfast BT3 9DT, UK \\ ${ }^{3}$ Department of Electrical and Computer Engineering, University of Cyprus, Nicosia, Cyprus
}

Correspondence should be addressed to Abdul Quddious; abdulquddious@gmail.com

Received 13 October 2017; Accepted 27 March 2018; Published 29 April 2018

Academic Editor: Ana Alejos

Copyright @ 2018 Abdul Quddious et al. This is an open access article distributed under the Creative Commons Attribution License, which permits unrestricted use, distribution, and reproduction in any medium, provided the original work is properly cited.

The use of a tunable power splitter (PS) as a constituent component of a simultaneous wireless information and power transfer (SWIPT) system is discussed. Two varactor diodes are used to achieve a tunable output power ratio $P_{2}: P_{3}$ varying from $1: 1$ to 1:10 under good matching conditions. The SWIPT system that operates at $2.4 \mathrm{GHz}$ consists of a typical patch antenna, cascaded with the tunable PS, and a voltage doubler rectifier. The constituent components were implemented and tested as stand-alone devices and were subsequently combined in a measurement system using interconnectors. The effect of the tunable PS was explored with respect to the SNR measurements on the port that is intended for the information decoding receiver and the DC voltage measurements on the termination load of the rectifier that is connected directly on the energy harvesting port of the tunable PS. A spectrum analyzer is used for the SNR measurements while the input power is controlled using a signal generator. Both wireless power transmission and on-board measurements verify that the harvested energy can be maximized by using the minimum SNR at the information decoding branch at the expense of DC power consumption required for the biasing of the varactor diodes.

\section{Introduction}

Simultaneous wireless information and power transfer (SWIPT) systems became appealing because of their prospective in prolonging the lifetime of energy-constrained wireless networks. Utilizing a radio signal to transfer energy as well as information has the potential of providing significant advantages to future communication systems, especially for the mobile users. The idea of transmitting an energy and information signal simultaneously was proposed for the first time in [1], where the assumption that a (smart) receiver would be able to harvest energy and decode the information signal simultaneously was made. The assumption did not have practical applications because energy harvesting circuits did not have the capability of decoding an information signal; therefore, two core solutions, namely, time switching and power splitting, were proposed and studied in detail in the following years [2]. The main difference between time switching and power splitting is that with time switching, the receiver performs either energy harvesting $(\mathrm{EH})$ or information decoding (ID) depending upon the precoder timeslot allocation. On the other hand, with power splitting, the RF signal received by an antenna is split into two signal streams, and one stream leads to the $\mathrm{EH}$ unit while the other one to the ID unit [3]. The applications of time switching and power splitting systems have been studied with a number of wireless systems mainly with orthogonal frequency division multiplexing (OFDM) systems [4, 5], multiple-input single-output (MISO) systems [6, 7], and multiple-input multiple-output (MIMO) systems [8]. Power splitting and time splitting approaches were studied on cooperative networks, and their throughputs and outage probability were 
analyzed in [9]. In [10, 11], game theory approaches were proposed for multiple source-destination pairs which communicate through their dedicated energy harvesting relays and relayed multiuser cooperative network, respectively (game theory approaches were proposed for a decodeand-forward (DF) and relayed multiuser cooperative network, resp.). In a more theoretical and mathematical approach, power splitting and time switching methods have been investigated and compared under different scenarios like the network-coded two-way networks in [12] or the MIMO-ODFM relay networks [13] where power splitting presented some advantages. In another occasion, improved effective transmission rate was achieved using hybrid protocols [14] utilizing a combination of the two concepts. From a more practical perspective, SWIPT systems with high-power on-demand electromagnetic radiation have also been discussed to potentially charge batteries of low-power sensor networks with high-energy constraints, like implantable biomedical sensors and smart wearable devices $[15,16]$, structure monitoring sensors [17], remotely located wireless nodes [18], and so on. It has been shown that the information transmission time and the spectral efficiency of power splitting-based SWIPT are better than the counterparts using time switchingbased SWIPT [11, 19]; however, the power loss associated with microwave power splitting systems has not been adequately discussed. The use of the Talbot effect for power splitting that was presented in [20] has limited throughput losses; nevertheless, it is difficult to be implemented for SWIPT systems. Another study investigated the arbitraryshaped discrete input signals considering peak and average power constraints and their impact on PS-SWIPT systems $[21,22]$, while a nontrivial trade-off has been pointed out between maximizing the information rate and maximizing the power rate in a transmission signal [23]. With the ongoing rapid interest in SWIPT systems, it is probable to segment future 5G communication, which aims to connect most devices in order to support the Internet of Everything (IoE). The current manuscript discusses the implementation of a tunable power splitter and its potential use for power splitting SWIPT systems though extensive experimental testing. The tunable power splitter (PS) uses two varactor diodes, and it was tested as a constituent component for a SWIPT experimental system. The experimental SWIPT system consists of a microstrip patch antenna, the tunable PS, and a voltage doubler rectifier. Three-port $S$ parameter measurements indicated that the proposed tunable PS could achieve wide $K=\left(P_{2}: P_{3}\right)$ values ranging from $1: 1$ to $1: 10$ with good matching at the three ports. The wide span of $K$ values was exploited to use the tunable PS as a constituent component in a SWIPT system in an attempt to maximize the harvested energy while simultaneously maintaining the minimum acceptable SNR at the information decoding receiver unit. Both wireless power measurements and on-board measurements verified that a tunable PS can control the received SNR at the information decoding receiver, and at the same time the harvested energy level on the $\mathrm{EH}$ unit, and therefore can potentially maximize the harvested energy of a SWIPT system while maintaining adequate SNR and the information decoder.

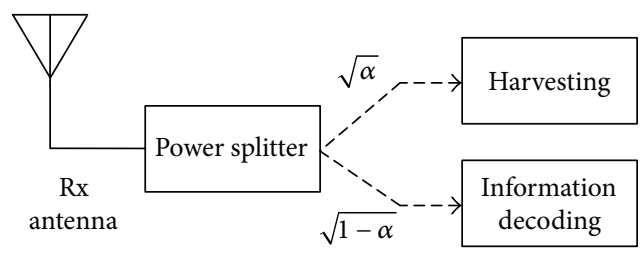

Figure 1: Schematic diagram of a SWIPT receiver using a power splitter (PS) component.

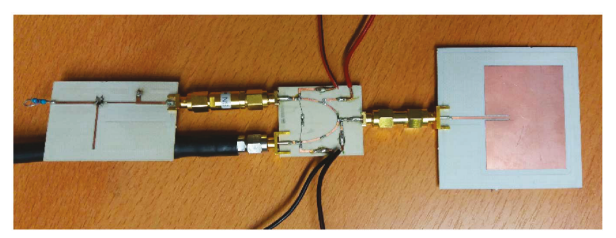

FIGURE 2: Implemented combination of the constituent modules for a SWIPT receiver for testing measurements.

\section{SWIPT Topology}

There are different protocols for simultaneous wireless information and power transfer (SWIPT) communications depending on the preferred method for separating the received signal: (a) time switching, for which the received power is connected either to the ID unit or to the energy harvesting (EH) unit; (b) spatial switching, for which a different antenna is used for the ID and a different one for the EH path; and (c) power splitting (PS), for which the single antenna is connected to a power splitter (or a power divider) and the two portions of the received power are directed to either the ID receiver or the energy harvester. Probably, the most popular method has been the use of a power splitter (PS). While, depending on the channel conditions, an optimum power splitting ratio can be theoretically calculated, these conditions are rarely constant. Therefore, often this ratio has to be changed for the SWIPT method to remain optimum. The power splitting technique requires higher receiver complexity and also the optimization of the power splitting factor $\alpha$; however, it achieves instantaneous wireless and power transfer, as the signal received in just one time slot is used for both information decoding and power transfer. Therefore, it is more suitable for applications with critical information/energy or delay constraints and closer to the information theoretical optimum [15]. Generally and considering that nowadays receivers have very high sensitivity (operate successfully for very low received signal power levels), effective communications can be established with fairly low signal to noise ratio (SNR). If the absolutely minimum power portion is used for the ID, the remaining maximized power can be allocated to the $\mathrm{EH}$ path for the rectification and exploitation of the RF input power. The SWIPT concept using a power splitter (PS) can be seen in the schematic representation of Figure 1. A typical system consists of a single antenna connected directly to a 3-port power splitter (or power divider). Port ${ }_{1}$ or the input port is the RF input power port, and ports Port ${ }_{2}$ and $\mathrm{Port}_{3}$ are the 


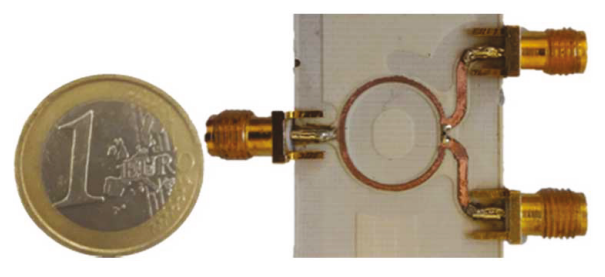

(a)

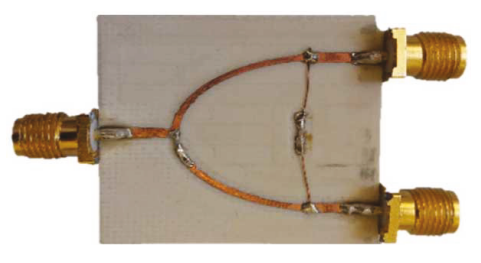

(b)

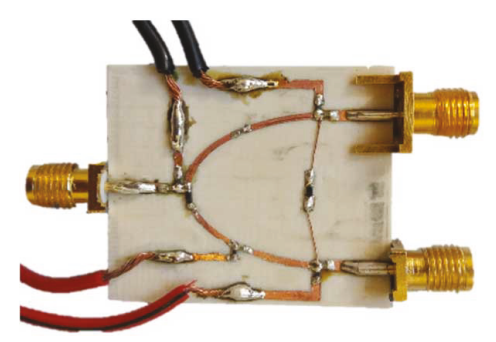

(c)

FIGURE 3: Implemented power splitters: (a) constant ratio-fixed characteristic impedances PS, (b) fixed lumped capacitor PS, and (c) tunable PS using varactor diodes.

output ports which are connected to either the $\mathrm{EH}$ unit or the ID module. For the two output ports and their related subsequent components, the figures of merit are different. The $\mathrm{EH}$ unit requires as large as possible power while the information decoder requires a large signal to noise ratio (SNR).

For the assessment of a SWIPT receiver under different power splitting conditions (different power ratios at the two output ports), the combined module presented in Figure 2 was used. It was designed to operate at the popular $2.4 \mathrm{GHz}$ band (used for WLAN applications), and it consists of a microstrip-fed rectangular patch antenna, a PS module, and a voltage doubler RF-to-DC rectifier. For SNR measurements, a spectrum analyzer (Anritsu MS2668C) was connected directly on one of the two output ports while the second output port was connected directly to the rectifier. The RF-to-DC rectifier was terminated with a $3.3 \mathrm{k} \Omega$ load, and the rectified voltage $V_{\mathrm{DC}}$ was measured across this $3.3 \mathrm{k} \Omega$ load. During testing, the receiver antenna and the rectifier were kept the same, while different PS modules were used. Figure 3 displays the three types of power splitters that were used: a Wilkinson divider using microstrip lines of different characteristic impedances, a divider with lumped capacitors, and a tunable power splitter using two varactor diodes. In order to investigate the effect of different power ratios under different biasing conditions on the performance of the SWIPT receiver, power splitters with different features were used, and their results were compared with the results of the tunable PS. $S$ parameter measurements presented in a subsequent section demonstrate that the tunable PS showed a good response for six different output power ratio combinations, namely, $1: 1,1: 2,1: 3,1: 4,1: 5$, and $1: 10$. The tunable PS achieved additional power ratios at the two output ports, ranging from $1: 16\left(P_{2}: P_{3}\right)$ up to $4: 1$ as they were measured using a spectrum analyzer although with varying percentage of loss. $P_{2}$ is the RF power measured at Port $_{2}$, and $P_{3}$ is the RF power measured at Port $_{3}$.

\section{RF-to-DC Rectifier}

The voltage doubler topology, which is preferred for its simplicity and fairly high efficiency, was used for the implementation of an RF-to-DC rectifier on a Rogers RO4003C substrate with $\varepsilon_{\mathrm{r}}=3.55$ and substrate thickness of $0.508 \mathrm{~mm}$. The voltage doubler topology consists of two diodes and two capacitors, and for the implemented prototype, two Skyworks Schotkky diodes SMS7630-079LF were used while the

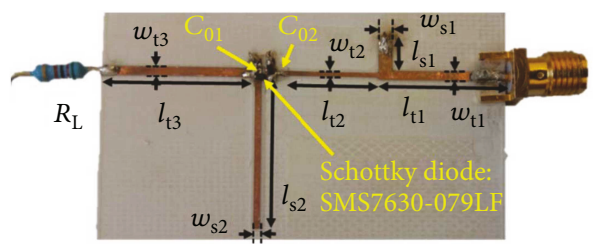

FIgURE 4: Fabricated $2.4 \mathrm{GHz}$ rectifier. All length dimensions are in $\mathrm{mm}: l_{\mathrm{t} 1}=16.56, l_{\mathrm{t} 2}=12.7, l_{\mathrm{t} 3}=19.2, \quad l_{\mathrm{s} 1}=4, \quad l_{\mathrm{s} 2}=19.5$, $w_{\mathrm{t} 1}=1.18, w_{\mathrm{t} 2}=0.59, w_{\mathrm{t} 3}=1.18, w_{\mathrm{s} 1}=1.76, w_{\mathrm{s} 2}=0.79, R_{\mathrm{L}}=3.3 \mathrm{k} \Omega$, $C_{01}=150 \mathrm{pF}$, and $C_{02}=150 \mathrm{pF}$.

capacitor values were $C_{01}=150 \mathrm{pF}$ and $C_{02}=150 \mathrm{pF}$. Figure 4 presents the fabricated prototype, and its detailed dimensions are listed in the caption. The most important design feature of a rectifier intended for energy harvesting is the high RF-to-DC efficiency calculated by (1) where $P_{\text {in }}$ and $P_{\text {out }}$ are the input RF power and the output DC power values in $(W)$, respectively, and $V_{\mathrm{DC}}$ is the voltage across the load resistance $R_{\mathrm{L}}$.

$$
\eta=\frac{P_{\text {out }}}{P_{\text {in }}}=\frac{V_{\mathrm{DC}}^{2} / R_{\mathrm{L}}}{P_{\text {in }}} .
$$

Designing a high-performance voltage doubler rectifier is a challenging task mainly because of the nonlinear nature of the diodes used. The rectifier's most important parameter is the efficiency $\eta$ that depends nonlinearly on the input power $P_{\text {in }}$ and on the termination load $R_{\mathrm{L}}$. The nonlinear dependency of the efficiency with respect to the aforementioned parameters is evident in the simulated results presented in Figure 5. Within the scope of the current manuscript, the design of the highest efficiency rectifier was not of paramount importance since the rectifier was terminated with an indicative termination load of $3.3 \mathrm{k} \Omega$, and there was no subsequent power management unit (PMU) which is usually necessary in order to preserve the optimum load for maximum efficiency conditions. On the other hand, good matching with the $50 \Omega$ characteristic impedance of the preceding power splitter was a strict requirement, and $S$ parameter measurements on the stand-alone device were taken to ensure the good matching condition. The measured and simulated reflection coefficient for the implemented rectifier is presented in Figure 6. For the $S$ parameter and rectified voltage measurements, the rectifier's input port was directly 


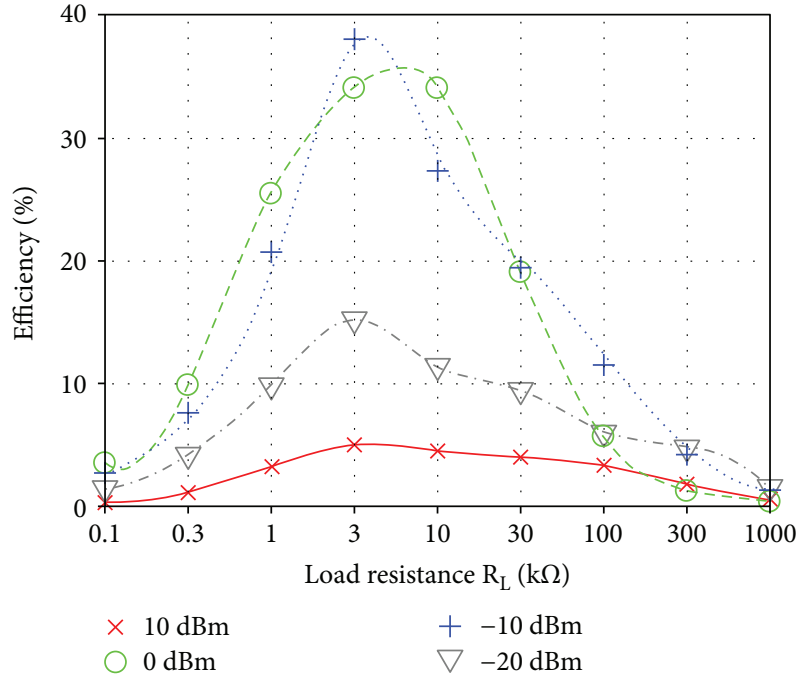

FIGURE 5: Simulated efficiency for different RF input power levels versus $R_{\mathrm{L}}$.

connected to an Agilent E8363B vector network analyzer (VNA), and an unmodulated sine wave was used as the $\mathrm{RF}$ input power. For the presented results in Figure 7, the input power was varied from -20 to $10 \mathrm{dBm}$ with a step size of $5 \mathrm{~dB}$. A digital multimeter (DMM) was used for the measurement of the rectified DC voltage across the selected termination load of $3.3 \mathrm{k} \Omega$ while the achieved RF-to-DC efficiency was calculated from the set of DC measurements using (1). The simulated and measured rectified voltage and the RF-to-DC efficiency are presented in Figures 7(a) and 7(b), respectively.

\section{Tunable Power Splitter (PS)}

4.1. Tunable Power Splitter Implementation. As mentioned in Section 2, three different types of power splitters (PS) were used for the experiments on the implemented combined module (Figure 2) SWIPT receiver. As a first step, a conventional Wilkinson divider using microstrip technology was implemented. The well-known topology is limited to small output ratios, $\left(K=\left(P_{2}: P_{3}\right)<4\right)$, because of the impractical narrow widths related with the required high-characteristic impedance lines. Therefore, the use of lumped capacitors was introduced. For the implemented lumped capacitor PS topology (simulation model presented in Figure $8(a)$ ), one capacitor was connected on one of the legs of the PS and the second one was connected along the bridge that connects the two legs. Based on this topology, the implementation of a tunable PS with two varactor diodes instead of the two lumped capacitors was achieved. For the DC biasing of the two varactors, DC biasing lines were needed, and because of the high DC voltage, three DC-blocking capacitors were integrated along each one of the three transmission lines that form the 3-port power splitter. Two more were used at the connection point of the biasing lines with the microstrip lines. Additionally, on the feed lines, RF choke inductors were used to prevent the RF signals from leaking into the feed

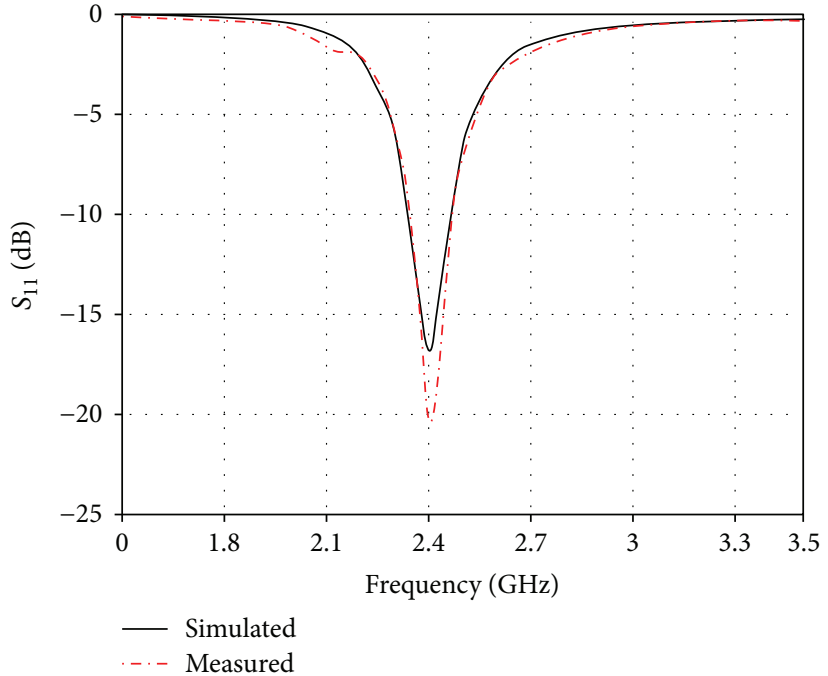

FIGURE 6: Simulated and measured reflection coefficient $\left(S_{11}\right)$ of the rectifier.

lines. The simulated tunable PS is presented in Figure 8(b), and the dimensions used for the prototype fabrication are listed in the caption. The varactors used (SMV2020-079LF) depending on the applied differential voltage (from 0-20 V) can have capacitances varying from 0.4 to $3.2 \mathrm{pF}$. The combinations of the varactor capacitances used give a large number of operational conditions, for which parameters such as the overall loss, the overall matching on all three ports, and the output power ratio $K=P_{2}: P_{3}$ need to be controlled. Using lumped capacitor components, six different modules with ratios $(1: 1,1: 2,1: 3,1: 4,1: 5$, and $1: 10)$ were implemented, and for the same ratios, the required varactor capacitances with the related biasing conditions are summarized in Table 1. By investigating all the possible varactor capacitances' combinations, the power ratio $P_{2}: P_{3}$ is plotted in Figure $9(\mathrm{a})$, and the power ratio $P_{3}: P_{2}$ is plotted in Figure 9(b), each as a two-dimensional graph. The resulted power ratio of $P_{2}: P_{3}$ spans the values from $1: 1$ up to $1: 16$, and for the power ratio $P_{3}: P_{2}$, the ratio can vary between $1: 1$ and $4: 1$.

4.2. S Parameter Measurements. Not all the biasing voltage combinations and the related varactor values result in acceptable loss and matching performance. However, for the set of the six different combinations mentioned above, $1: 1,1: 2$, $1: 3,1: 4,1: 5$, and $1: 10, S$ parameter measurements were taken, and they were compared with the six fabricated modules for which lumped capacitors were used. The starting values for the varactor capacitors were the values of the corresponding lumped capacitors, but additional tuning was required since the addition of the RF choke inductors and the DC-blocking capacitors caused perturbations. As a result, the presented $S$ parameter measurements conducted with a two-port VNA while the third port was terminated with a $50 \Omega$ load to eliminate reflections present some differences when the measured $S$ parameters of the lumped component PS are compared with the measured $S$ parameters of the 


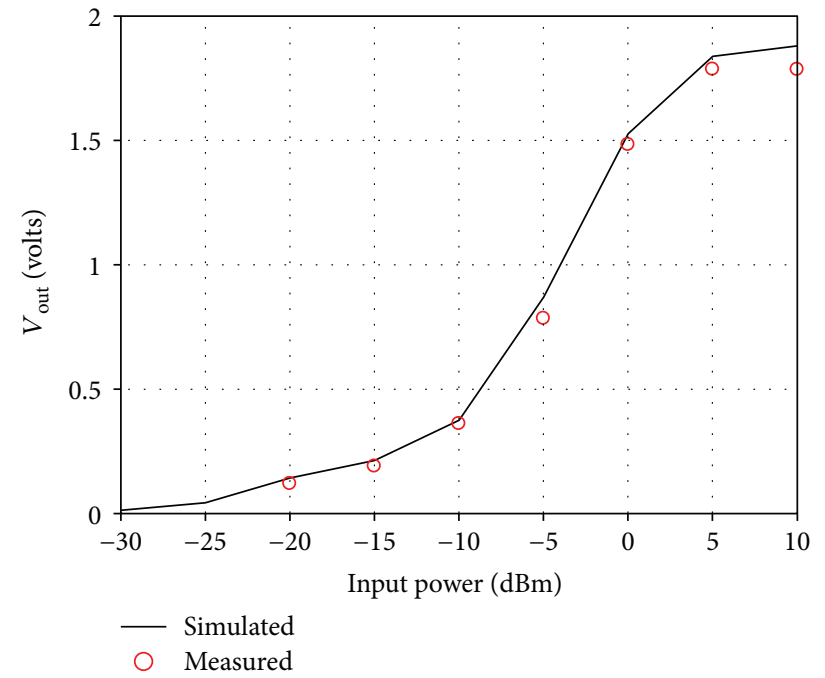

(a)

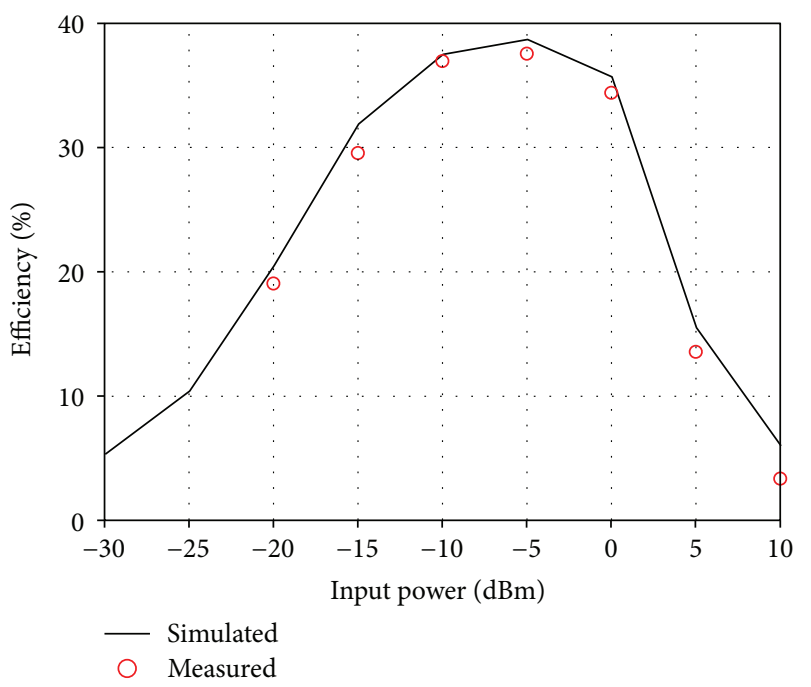

(b)

FIGURE 7: (a) Rectified DC voltage versus RF input power simulations and measurements. (b) RF-to-DC efficiency versus RF input power simulations and measurements.

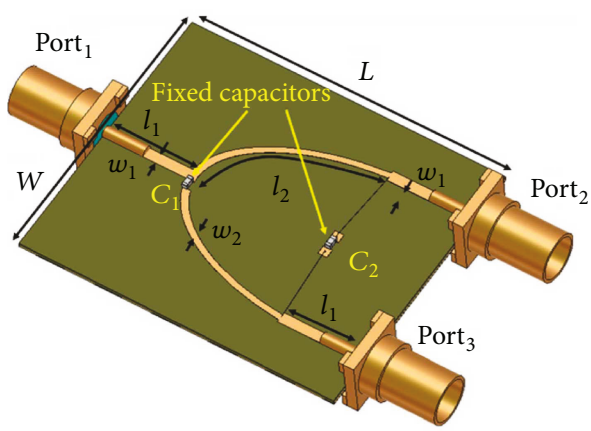

(a)

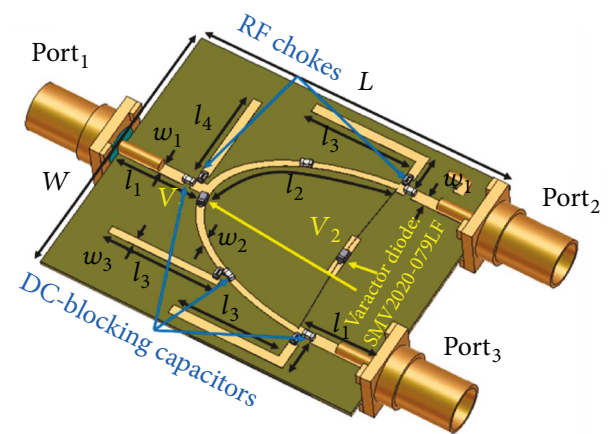

(b)

FIGURE 8: Full-wave EM simulation module of the fabricated (a) fixed ratio lumped capacitors PS and (b) tunable PS using two varactor diodes; all dimensions are in $\mathrm{mm}: L=35.5, W=30, l_{1}=7.7, l_{2}=19.5, l_{3}=12, l_{4}=10, w_{1}=1.18, w_{2}=0.9, w_{3}=1, C_{1}=\mathrm{pF}$, and $C_{2}=\mathrm{pF}$.

TABle 1

\begin{tabular}{lcccc}
\hline \multirow{2}{*}{ Power ratios } & \multicolumn{2}{c}{ Capacitance $(\mathrm{pF})$} & \multicolumn{2}{c}{ DC biasing voltage (V) } \\
& Varactor 1 & Varactor 2 & Varactor 1 & Varactor 2 \\
\hline $1: 1$ & 3.2 & 3.2 & 0 & 0 \\
$1: 2$ & 1.0 & 0.45 & 6 & 13 \\
$1: 3$ & 0.45 & 1.35 & 13 & 4 \\
$1: 4$ & 0.45 & 0.65 & 13 & 9 \\
$1: 5$ & 0.35 & 1.75 & 20 & 2.5 \\
$1: 10$ & 0.20 & 1.1 & 20 & 6 \\
\hline
\end{tabular}

tunable PS. Six $S$ parameter plots, one for each of the six fabricated fixed lumped component PS, are presented in Figure 10. For better clarity, only the magnitudes of $S_{11}, S_{31}$, and $S_{21}$ are presented to show the good matching at Port ${ }_{1}$, and the output power ratio $\left(K=P_{3}: P_{2}\right)$ as it can be derived indirectly from $S_{31}$ and $S_{21}$ indications, and can be calculated after some mathematical processing. Despite the complicated implementation of the tunable PS, with the necessity for DCblocking capacitors and RF choke inductors, its $S$ parameter measurements agree fairly well with the measurements for the fixed ratio, lumped capacitor PS.

4.3. SNR Measurements. As discussed briefly in Section 2, while for the $\mathrm{EH}$ path, it is important to have a high power, and for the information decoding path, the most important parameter is the SNR at the input of the information decoder receiver. Therefore, in combination with the harvested power at the end of the rectifier, a spectrum analyzer (Anritsu MS2668C) was used to measure the SNR at the output of the PS. The measurement setup is presented in Figure 11. The implemented tunable PS is fed with the RF input power generated from a signal generator (Rohde \& Schwarz SMF100A) through Port ${ }_{1}$. At the two output ports, namely, Port $_{2}$ and Port $_{3}$, the rectifier module was connected using connectors (Figure 12), and a spectrum analyzer was connected directly to the tunable PS for power measurements and SNR measurements. For several different power ratio 


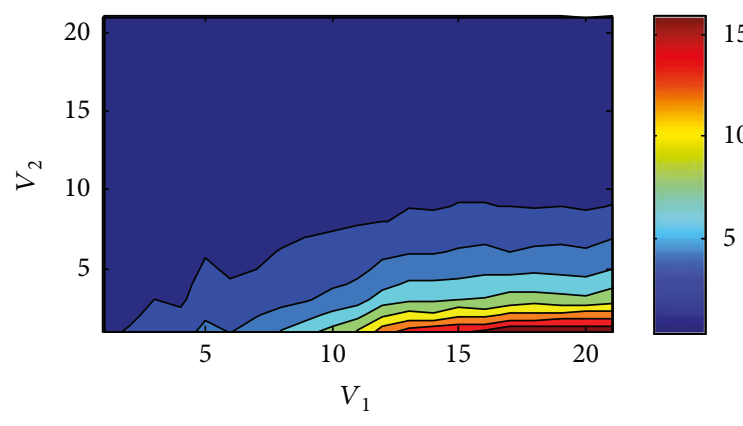

(a)

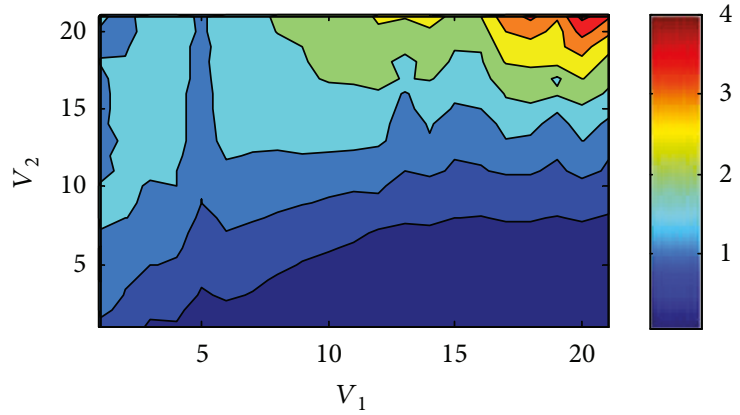

(b)

FIGURE 9: All DC biasing voltage combinations for the resulting power ratios for (a) $P_{2}: P_{3}(1: 1-1: 16)$ and (b) $P_{3}: P_{2}(1: 1-4: 1)$.

combinations, the measurements were repeated while the ports connected to the rectifier and the spectrum analyzer were interchanged. The measurements for a setup where Port $_{2}$ was connected to the rectifier and $\mathrm{Port}_{3}$ was connected to the spectrum analyzer are presented in Figure 12. At the same time when the rectified voltage across the $3.3 \mathrm{k} \Omega$ load was measured, the $\mathrm{SNR}$ at Port ${ }_{3}$ was also measured. Since the rectifier is a nonlinear circuit, three sets of measurements were taken for different $\mathrm{RF}$ input power levels. The power levels at the signal generator were $-10 \mathrm{dBm}, 0 \mathrm{dBm}$, and $+10 \mathrm{dBm}$. Before connecting the cable to the tunable PS, it was connected to the spectrum analyzer, and the available power (after the cable losses) at the input of the tunable PS was measured as a manual calibration. For the previous set of measurements, the rectifier was connected to the "low-power" leg ending at Port $_{2}$. As a result, as the power ratio $P_{2}: P_{3}$ decreased from $1: 1$ to $1: 10$, the rectified voltage also decreased. At the same time since more power was made available at the information decoding port ("high-power" Port $_{3}$ ), with the noise remaining stable, the measured SNR on $\mathrm{Port}_{3}$ increased nonlinearly. Figure 13 verifies the observations. When the same measurements were repeated (signal generator power set to $0 \mathrm{dBm}$ ) with the rectifier connected to the "high-power" Port $_{3}$, the rectified voltage increased as the power ratio $K=P_{2}: P_{3}$ decreased, since more power was made available at Port ${ }_{3}$. Apparently, the measured SNR at "low-power" Port ${ }_{2}$ decreased. The two measurement setups with the rectifier connected to the "low-power" Port 2 (Figure 14(a)) and the rectifier connected to the "high-power" $\mathrm{Port}_{3}$ (Figure 14(b)) are presented in Figure 14.

\subsection{Overall Loss and DC Power Consumption Considerations.} For the presented $S$ parameter measurements, it can be observed that there is good matching, accurate power ratios, and relatively low loss. However, since there are many combinations of the varactor biasing values $V_{1}$ and $V_{2}$ that may cause the same power ratio, unavoidably the loss and the induced noise are different and they need to be carefully considered. For the calculation of losses on the 3-port tunable PS, the power levels at Port 1 , Port 2 , and Port 3 were measured using the spectrum analyzer. For each output port power measurement, the second output port was terminated with a $50 \Omega$ load. Measurements were taken for all varactor capacitance combinations that caused power ratio $1: 1$. Figure 15 presents in color the DC biasing condition combinations for which the power ratio is $1: 1$. The color scale indicates the loss as a percentage normalized over the incident power $P_{1}$ at Port ${ }_{1}$. The formula for the presented loss percentage is given by

$$
\text { Loss }=\frac{P_{1}-\left(P_{2}-P_{3}\right)}{P_{1}} \%
$$

For some biasing conditions and their resulting capacitance combinations, the loss was unacceptably high; therefore, the preferred combination should be chosen based on the total loss and also on the associated DC power consumption required for the varactors' biasing conditions.

In addition to the voltage values for a given capacitance mentioned in the data sheet, measurements were taken for many more biasing voltage combinations for each varactor diode from 0 to $21 \mathrm{~V}$. It was observed that several specific power ratios could be obtained using a set of pairs of biasing voltages instead of just one. In such a case, the choice of the most suitable biasing conditions could be either (a) the minimum DC power consumption for the specific power ratio or (b) the minimum overall device loss, when the specific power ratio was achieved. Figure 16(a) shows a useful plot for the choice of biasing voltages while Figure 16(b) demonstrates the overall device loss percentage with the associated biasing conditions. In Figure 16(b), it can be noticed that the overall device loss is rather high for extremely high and/or low biasing voltages (red-circled areas), while the device operates with lower loss for low $V_{1}(<12 \mathrm{~V})$ and high $V_{2}(>9 \mathrm{~V})$ values (blue area).

4.5. Added Noise. Finally, in order to estimate the introduced noise from the lumped components which are included in the lumped capacitor PS module with power ratio $1: 1$ that affects the SNR on the information decoding path, rectified voltage and SNR measurements were taken when the rectifier and the spectrum analyzer are interchanged between the two output ports. The results were expected to be very similar; however, the measurements which are presented in Figure 17 indicate that while the rectified power remained the same when the rectifier was connected to either port, 


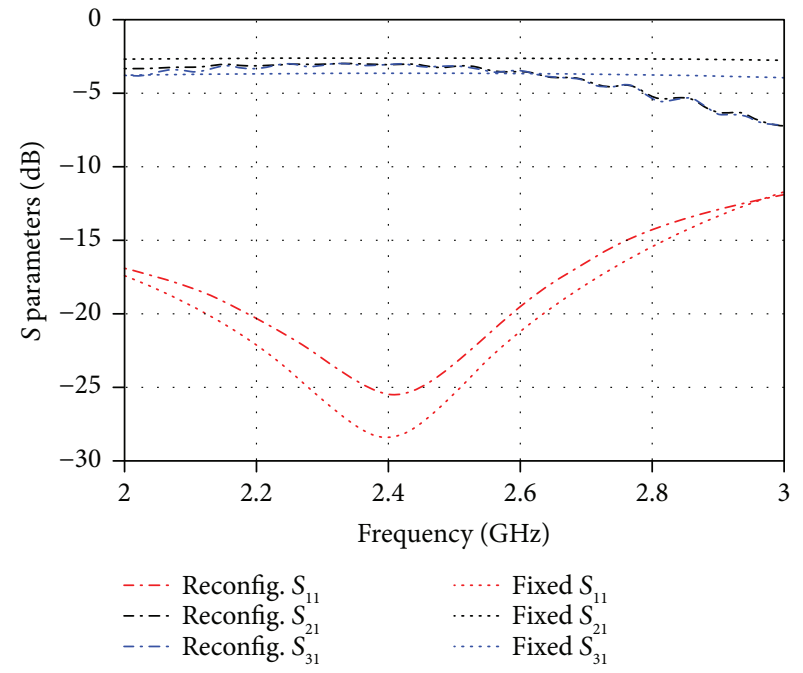

(a) $1: 1$

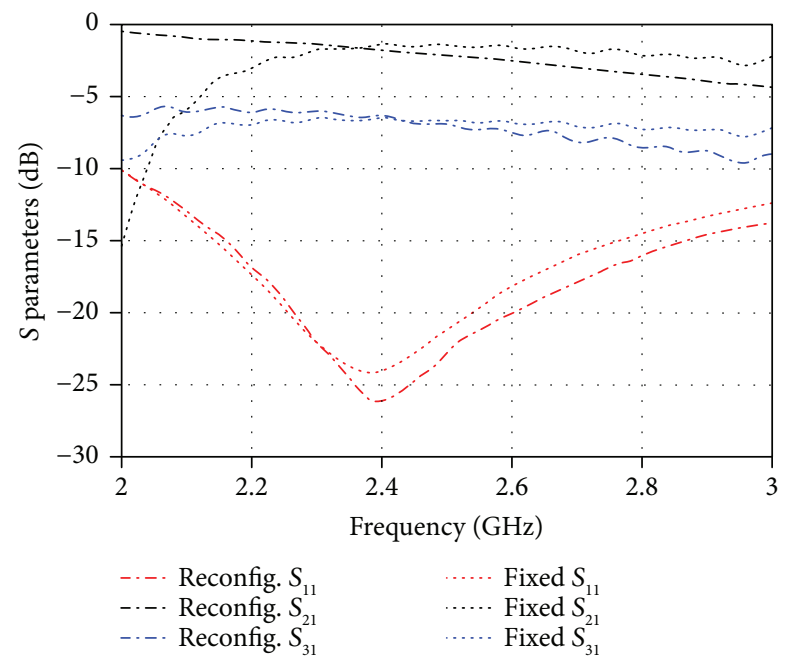

(c) $1: 3$

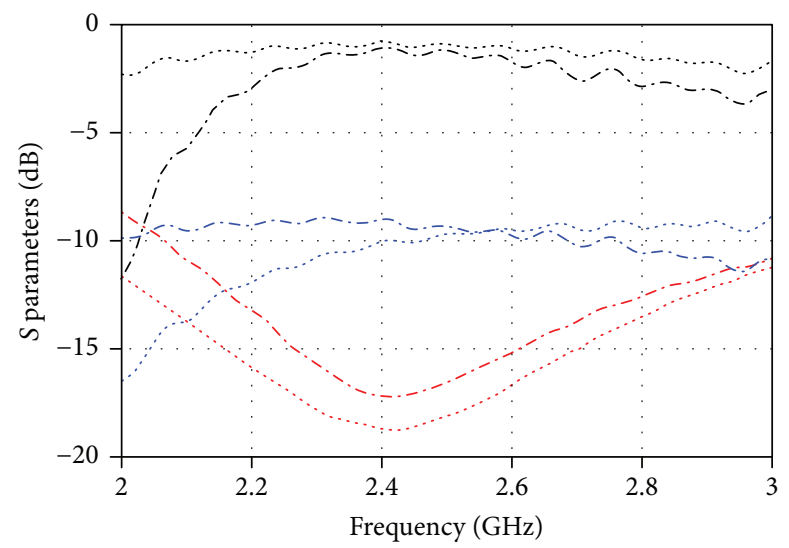

$\begin{array}{ll}\text {-.-. Reconfig. } S_{11} & \ldots . . \text { Fixed } S_{11} \\ \text {-.-. Reconfig. } S_{21} & \ldots \ldots \text { Fixed } S_{21} \\ \text {-.-. Reconfig. } S_{31} & \ldots \cdots \text {. Fixed } S_{31}\end{array}$

(e) $1: 5$

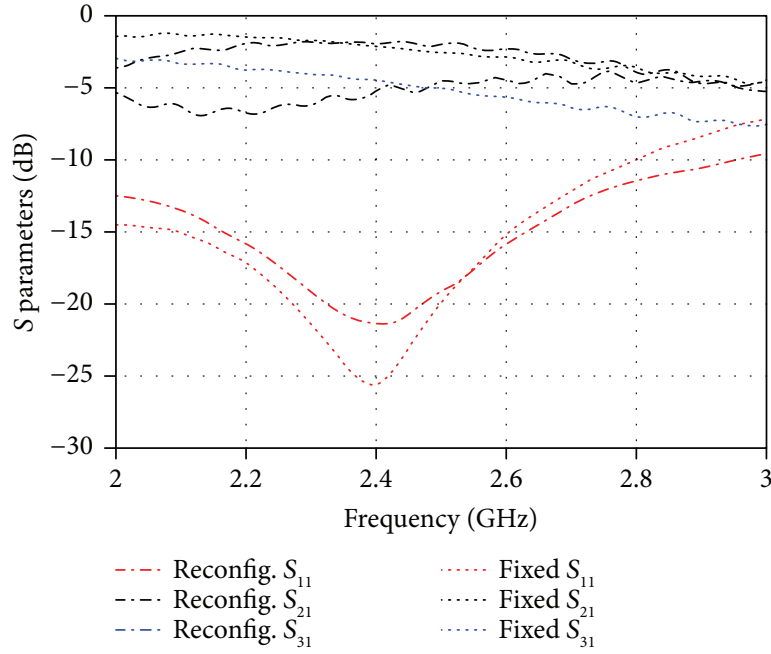

(b) $1: 2$

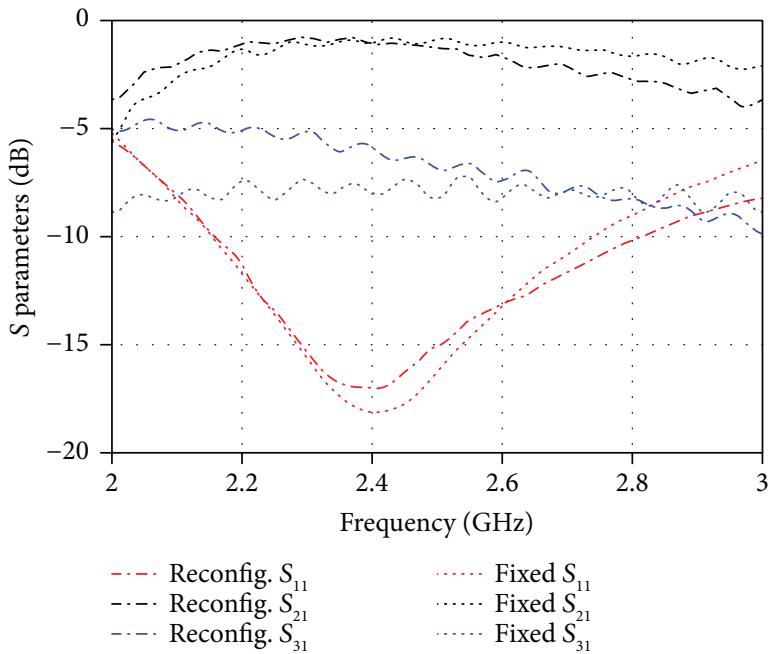

(d) $1: 4$
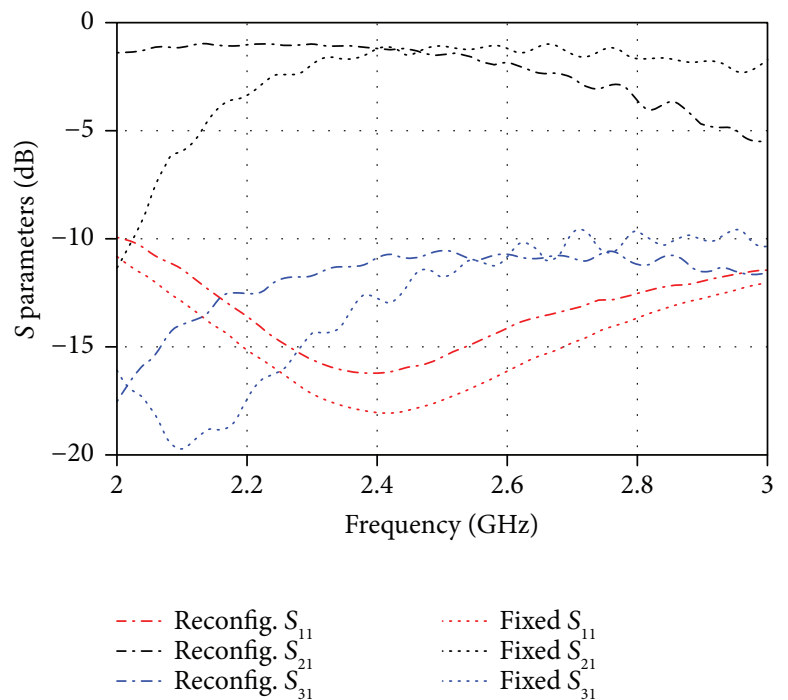

(f) $1: 10$

FIGURE 10: Measured $S$ parameters for lumped capacitor PS and tunable PS using varactor diodes for different power ratios: (a) $1: 1$, (b) $1: 2$, (c) $1: 3$, (d) $1: 4$, (e) $1: 5$, and (f) $1: 10$. 


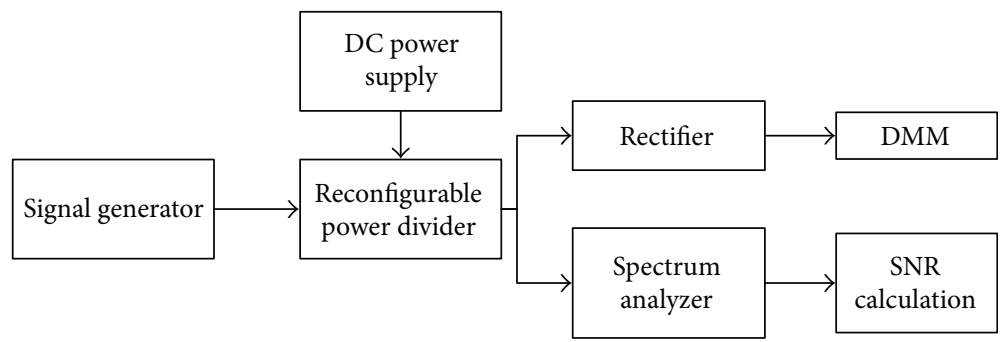

FIGURE 11: Conceptual schematic of the experimental setup.

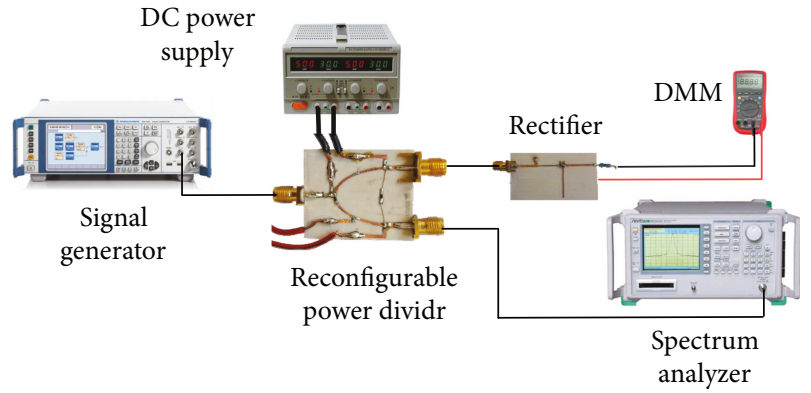

Figure 12: Experimental setup for the validation of the implemented SWIPT receiver.

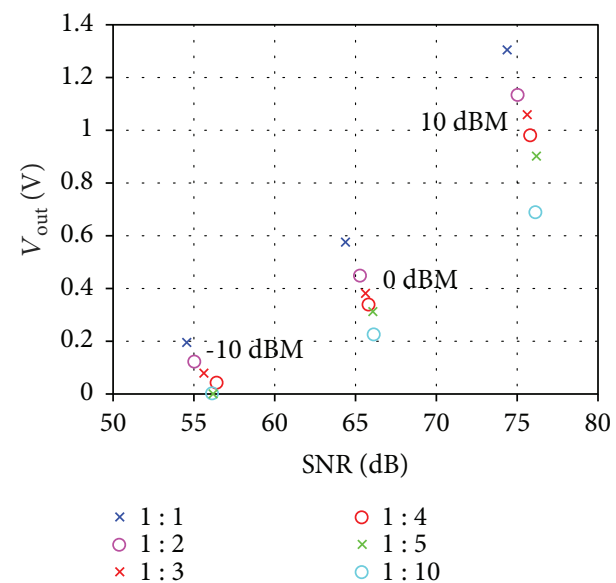

FIGURE 13: Measured rectified voltage $V_{\text {out }}$ at the output of the rectifier which was connected to Port ${ }_{2}$ versus the measured SNR at $\mathrm{Port}_{3}$, for three different power levels controlled at the signal generator: $-10 \mathrm{dBm}, 0 \mathrm{dBm}$, and $+10 \mathrm{dBm}$.

the SNR degraded significantly when it was connected on the path that included the lumped capacitor $\left(\right.$ Port $\left._{2}\right)$. Figure 17(a) that presents the measured power at both Port 2 and Port shows identical behavior between the two cases. In Figure 17(b) though, where the measured SNR is presented, it is evident that the lumped components, which are necessary for the tunability of the power ratio, introduced additional noise that degraded the SNR at the input of the information decoding system. Consequently, when possible, the noisy path should be occupied by the $\mathrm{EH}$ circuit to ensure improved SNR along the ID path.

\section{Combined Module Testing}

For the combined module (antenna + tunable PS + rectifier), an inset-fed microstrip rectangular patch antenna [24] on a Rogers RO4003C, $0.508 \mathrm{~mm}$ thick substrate with $\varepsilon_{\mathrm{r}}=3.5$ and $\tan \delta=0.0027$, was fabricated. The antenna has a $70 \%$ radiation efficiency and a $4.86 \mathrm{dBi}$ simulated realized gain at $2.4 \mathrm{GHz}$. The photo of the fabricated prototype is presented in Figure 18(a), and its dimensions are listed in the caption. Simulated and measured reflection coefficient plots are presented in Figure 18(b). For the actual wireless power transmission measurement, two rectangular patch antennas were used in the laboratory setup presented in Figure 19(a). The first was connected to a signal generator and acted as the transmitter, and the second was connected to a spectrum analyzer and was used as the receiver. For different transmission power levels and for different separation distances, the received power at the antenna was recorded. This received power is expected to be the input power at the tunable PS neglecting the small mismatch. For those measured received power levels, the delivered power $P_{2}$ (at Port ${ }_{2}$ of the tunable PS) and the rectified voltage at the termination load were measured as shown in Figure 19(b). The measured results are summarized in Table 2, for the lowest DC power consumption varactor combination and for a $1: 1$ ratio, something that verifies the successful implementation of the tested WPT system. The resulting rectified voltages and the resulted SNR measurements for a SWIPT system that used a tunable PS can be found in Table 2 .

\section{Conclusion}

A tunable power splitter (PS) using two varactor diodes was designed and fabricated and was tested as a constituent component for a SWIPT experimental system that consists of a microstrip patch antenna, the tunable PS, and a voltage doubler rectifier. Both the microstrip patch antenna and the rectifier were built and tested as stand-alone devices, and they were combined in a SWIPT system for testing measurements using interconnectors. Three-port $S$ parameter measurements indicated that the proposed tunable PS could achieve wide $K=\left(P_{2}: P_{3}\right)$ values ranging from $1: 1$ to $1: 10$ with good matching at the three ports. Additional power measurements 


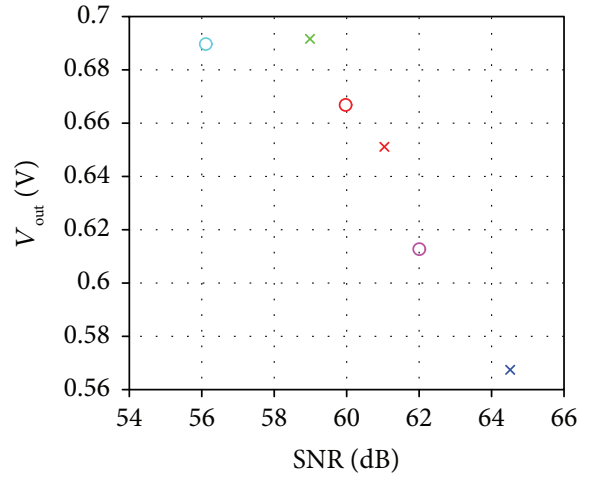

$\begin{array}{ll}\times 1: 1 & \circ 1: 4 \\ \circ 1: 2 & \times 1: 5 \\ \times 1: 3 & \circ 1: 10\end{array}$

(a)

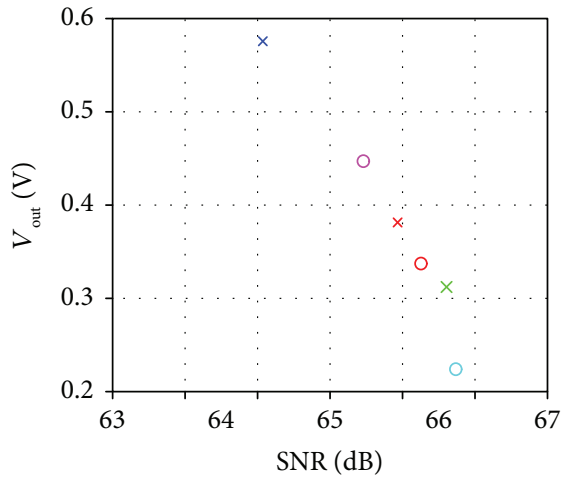
$\times 1: 1$
- $1: 4$
- $1: 2$
$\times 1: 5$
- $1: 10$

(b)

FIgURE 14: Measured rectified voltage $V_{\text {out }}$ at the output of the rectifier which was connected to Port versus the measured SNR at Port $_{3}$ for the six power ratios $(1: 1,1: 2,1: 3,1: 4,1: 5$, and $1: 10)$ when (a) the "high-power" leg is connected to the rectifier and (b) the "low-power leg" is connected to the rectifier, while the input power at Port ${ }_{1}$ is $0 \mathrm{dBm}$.

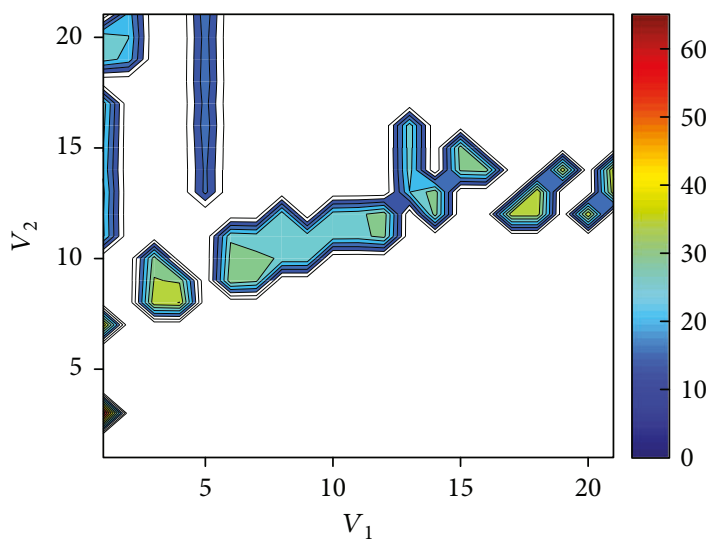

FIgURE 15: Loss percentage for varactor biasing conditions that cause power ratio $P_{2}: P_{3}=1: 1$.

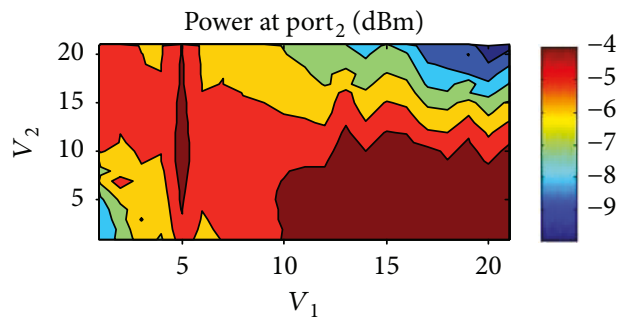

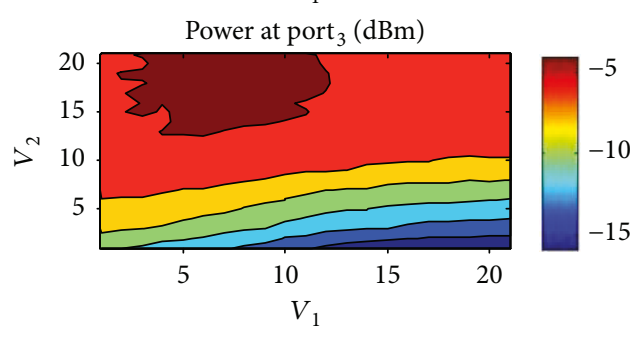

(a)

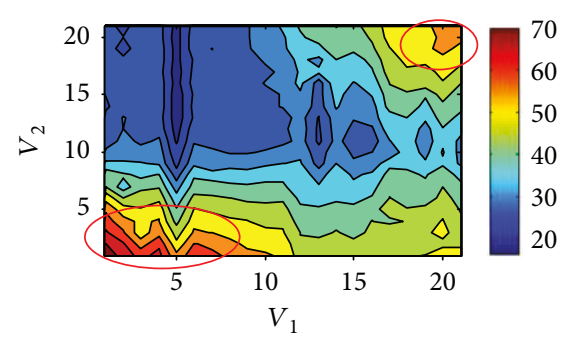

(b)

FIGURE 16: (a) Measured output power in $\mathrm{dBm}$ for both output ports of the tunable PS. While the measurement was taken on one port using the spectrum analyzer, the other port was connected to a $50 \Omega$ wideband load. The horizontal and vertical axes show the varactor diodes' biasing voltages. (b) Calculated overall loss of the tunable PS for biasing voltage conditions ranging from 0 to $20 \mathrm{~V}$, for both varactor diodes. 


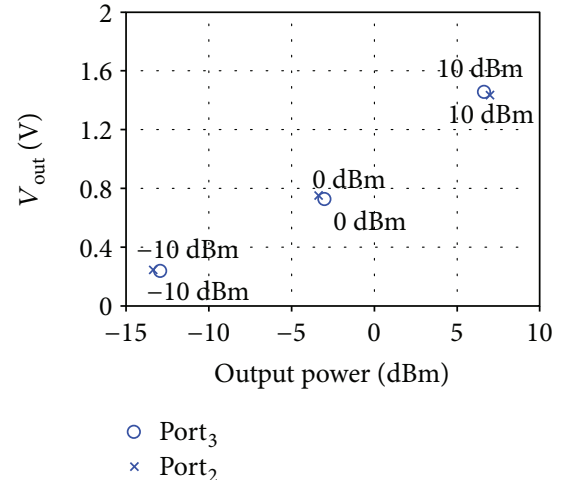

(a)

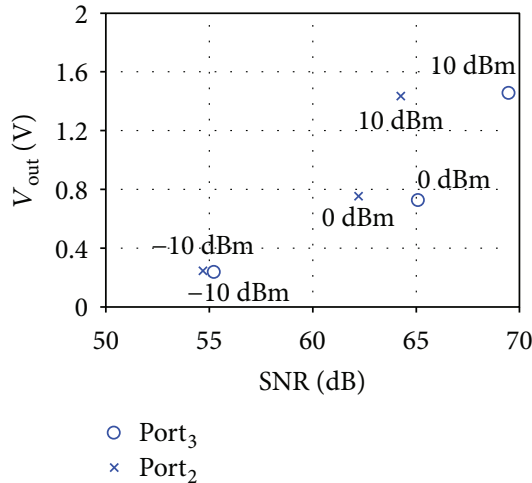

(b)

FIGURE 17: (a) $V_{\text {out }}$ versus output power and (b) $V_{\text {out }}$ versus SNR showing the effect of lumped components on resulting SNR.
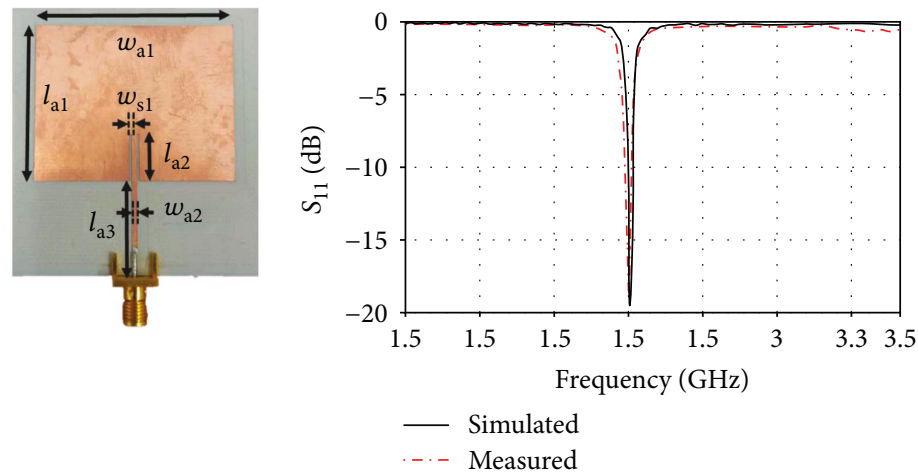

(a)

(b)

FIGURE 18: (a) Fabricated patch antenna with all dimensions in mm: $l_{\mathrm{a} 1}=35, l_{\mathrm{a} 2}=11, l_{\mathrm{a} 3}=17.5, w_{\mathrm{a} 1}=45, w_{\mathrm{a} 2}=1.14$, and $w_{\mathrm{s} 1}=0.47$, and $(\mathrm{b})$ simulated and measured patch antenna reflection coefficient.

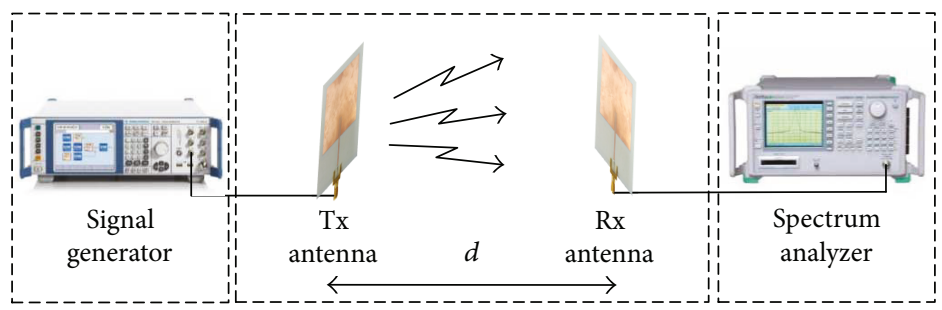

(a)

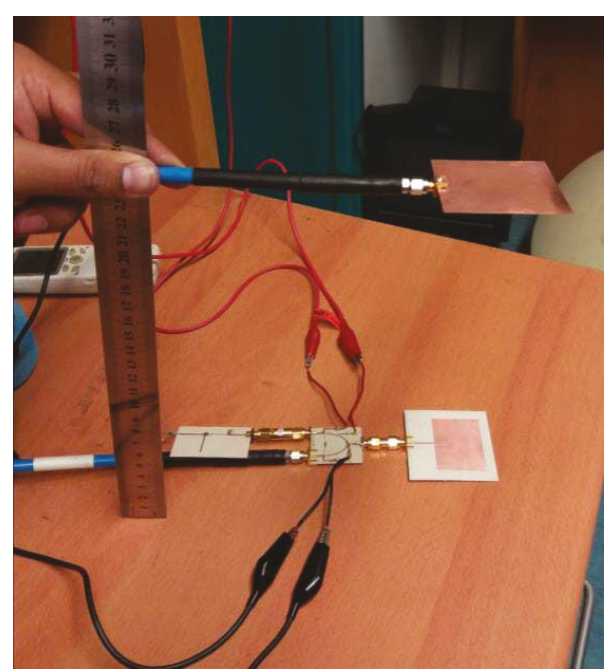

(b)

FIgURE 19: (a) Measurement setup to define the power at the input of the PS and (b) laboratory setup.

using a spectrum analyzer showed that even higher ratios up to $1: 16$ could be achieved with the proposed topology, and in addition, Port $_{2}$ could be made the high-power port with measured ratios $\left(P_{3}: P_{2}\right)$ from $1: 1$ to $4: 1$. The wide span of $K$ values was exploited to use the tunable PS as a constituent component in a SWIPT system in an attempt to maximize 
TABLE 2

\begin{tabular}{lccccc}
\hline \multicolumn{5}{c}{ Signal generator power: $15 \mathrm{dBm}$} \\
Distance $(\mathrm{m})$ & 0.1 & 0.2 & 0.3 & 0.4 & 0.5 \\
$P_{\mathrm{Rx}}(\mathrm{dBm})$ & -2.38 & -5.27 & -8.12 & -10.03 & -11.19 \\
$V_{\text {out }}(\mathrm{V})$ & 0.625 & 0.352 & 0.255 & 0.215 & 0.195 \\
$\mathrm{SNR}(\mathrm{dB})$ & 63.1 & 60.3 & 58.6 & 55.5 & 54.3 \\
\hline \multicolumn{5}{c}{ Signal generator power: $10 \mathrm{dBm}$} \\
Distance $(\mathrm{m})$ & 0.1 & 0.20 & 0.3 & 0.4 & 0.35 \\
$P_{\mathrm{Rx}}(\mathrm{dBm})$ & -0.85 & -7.49 & -10.24 & -13.51 & -15.37 \\
$V_{\text {out }}(\mathrm{V})$ & 0.751 & 0.312 & 0.205 & 0.155 & 0.145 \\
SNR $(\mathrm{dB})$ & 64.8 & 58.2 & 55.56 & 52.4 & 50.3 \\
\hline & Signal generator & power: $5 \mathrm{dBm}$ \\
Distance $(\mathrm{m})$ & 0.1 & 0.15 & 0.2 & 0.25 & 0.25 \\
$P_{\mathrm{Rx}}(\mathrm{dBm})$ & -6.35 & -11.82 & -15.15 & -18.09 & -20.07 \\
$V_{\text {out }}(\mathrm{V})$ & 0.325 & 0.205 & 0.145 & 0.085 & 0.055 \\
SNR $(\mathrm{dB})$ & 59.3 & 53.9 & 50.6 & 47.8 & 45.7 \\
\hline & Signal generator & power: $0 \mathrm{dBm}$ \\
Distance $(\mathrm{m})$ & 0.05 & 0.10 & 0.15 & 0.20 & 0.25 \\
$P_{\mathrm{Rx}}(\mathrm{dBm})$ & -5.23 & -11.15 & -14.35 & -16.89 & -18.67 \\
$V_{\text {out }}(\mathrm{V})$ & 0.355 & 0.212 & 0.165 & 0.128 & 0.075 \\
SNR $(\mathrm{dB})$ & 60.4 & 54.6 & 51.5 & 49.1 & 47.2 \\
\hline
\end{tabular}

the harvested energy while simultaneously maintaining the minimum acceptable SNR at the information decoding receiver unit. A series of measurements verified that the SNR and the rectified voltage improved when most of the incident input power was directed to the information decoding unit or the energy harvester, respectively. Since several power ratios could be achieved with more than one pair of voltages on the two varactors, the preferred biasing conditions could be chosen in order to satisfy both, or either, of the following objectives: either minimum DC power consumption (used for the varactor biasing) or minimum overall loss of the tunable PS. Using the lumped capacitor PS with a ratio of $1: 1$, a series of SNR measurements and rectified voltage measurements on both output ports, interchangeably, indicated that the lumped components used for tunability added noise to the signal that passed through them. Therefore, it is preferred to connect the energy harvester on the port that terminates the path with the noisy lumped components, in order to ensure improved SNR at the information decoding receiver path. For the wireless power transmission measurements, two microstrip patch antennas were used: one connected with a signal generator acting as the transmitter and the second one connected to a spectrum analyzer to measure directly the received power that was made available at the input of the tunable PS. Both wireless power measurements and on-board measurements verified that a tunable PS can control and therefore tune the received SNR at the information decoding receiver and the harvested energy level on the EH unit at the expense of DC power consumption used for biasing and some overall loss increase, which can be limited by choosing the most suitable biasing conditions. In future work, the following topics will be investigated: the size reduction of the tunable PS, the reduction of the DC power consumption on the switches, and the integration of the constituent modules into a compact single module. Furthermore, the implementation of similar integrated modules for UHF frequency will be investigated under the considerations of a desired compact size system.

\section{Conflicts of Interest}

The authors declare that they have no conflicts of interest.

\section{References}

[1] L. R. Varshney, "Transporting information and energy simultaneously," in 2008 IEEE International Symposium on Information Theory, pp. 1612-1616, Toronto, ON, Canada, July 2008.

[2] R. Jiang, K. Xiong, Y. Zhang, and Z. Zhong, "Outage analysis and optimization of SWIPT in network-coded two-way relay networks," Mobile Information Systems, vol. 2017, Article ID 2516035, 16 pages, 2017.

[3] S. Li, X. Zhou, C.-X. Wang, D. Yuan, and W. Zhang, "Joint transmit power allocation and splitting for SWIPT aided OFDM-IDMA in wireless sensor networks," Sensors, vol. 17, no. 7, p. 1566, 2017.

[4] J. Wu, W. Lu, H. Peng, X. Liu, and J. Hua, "Simultaneous wireless information and power transfer in OFDM systems based on subcarrier allocation," in 2016 International Wireless Communications and Mobile Computing Conference (IWCMC), pp. 926-929, Paphos, Cyprus, September 2016.

[5] X. Di, K. Xiong, Y. Zhang, and Z. Qiu, "Simultaneous wireless information and power transfer in two-hop OFDM decodeand-forward relay networks," KSII Transactions on Internet and Information Systems, vol. 10, no. 1, pp. 152-167, 2016.

[6] Z. Zhu, Z. Wang, X. Gui, and X. Gao, "Robust downlink beamforming and power splitting design in multiuser MISO SWIPT system," in 2014 IEEE/CIC International Conference on Communications in China (ICCC), pp. 271-275, Shanghai, China, October 2014.

[7] M. R. A. Khandaker and K.-K. Wong, "SWIPT in MISO multicasting systems," IEEE Wireless Communications Letters, vol. 3, no. 3, pp. 277-280, 2014.

[8] Z. Zong, H. Feng, S. Zhang, T. Yang, and B. Hu, "Joint transceiver design for simultaneous wireless information and power transfer in multi-user MIMO interference networks," in 2014 Sixth International Conference on Wireless Communications and Signal Processing (WCSP), pp. 1-6, Hefei, China, October 2014.

[9] P. Liu, S. Gazor, I.-M. Kim, and D. I. Kim, "Energy harvesting noncoherent cooperative communications," IEEE Transactions on Wireless Communications, vol. 14, no. 12, pp. $6722-$ 6737, 2015.

[10] B. Fang, W. Zhong, S. Jin, Z. Qian, and W. Shao, "Game-theoretic precoding for SWIPT in the DF-based MIMO relay networks," IEEE Transactions on Vehicular Technology, vol. 65, no. 9, pp. 6940-6948, 2016.

[11] H. Chen, Y. Li, Y. Jiang, Y. Ma, and B. Vucetic, "Distributed power splitting for SWIPT in relay interference channels using game theory," IEEE Transactions on Wireless Communications, vol. 14, no. 1, pp. 410-420, 2015.

[12] K. Xiong, Y. Zhang, Y. Chen, and X. Di, "Power splitting based SWIPT in network-coded two-way networks with data rate 
fairness: an information-theoretic perspective," China Communications, vol. 13, no. 12, pp. 107-119, 2016.

[13] K. Xiong, P. Fan, C. Zhang, and K. B. Letaief, "Wireless information and energy transfer for two-hop non-regenerative MIMO-OFDM relay networks," IEEE Journal on Selected Areas in Communications, vol. 33, no. 8, pp. 1-1611, 2015.

[14] V. Singh and H. Ochiai, "An efficient time switching protocol with adaptive power splitting for wireless energy harvesting relay networks," in 2017 IEEE 85th Vehicular Technology Conference (VTC Spring), pp. 1-5, Sydney, NSW, Australia, June 2017.

[15] I. Krikidis, S. Timotheou, S. Nikolaou, G. Zheng, D. W. K. Ng, and R. Schober, "Simultaneous wireless information and power transfer in modern communication systems," IEEE Communications Magazine, vol. 52, no. 11, pp. 104-110, 2014.

[16] L. Wang, F. Hu, Z. Ling, and B. Wang, "Wireless information and power transfer to maximize information throughput in WBAN," IEEE Internet of Things Journal, vol. 4, no. 5, pp. 1663-1670, 2017.

[17] Z. Zong, H. Feng, F. R. Yu, N. Zhao, T. Yang, and B. Hu, "Optimal transceiver design for SWIPT in K-user MIMO interference channels," IEEE Transactions on Wireless Communications, vol. 15, no. 1, pp. 430-445, 2016.

[18] A. A. ElSabaa, M. Patwary, E. Benkhelifa, A. Zaki, and S. E. ElKhamy, "On consumable energy allocation frame-work with SWIPT for remotely located wireless node within internet of things," in 2017 Second International Conference on Fog and Mobile Edge Computing (FMEC), pp. 201-206, Valencia, Spain, May 2017.

[19] H. Liu, K. J. Kim, K. S. Kwak, and H. Vincent Poor, "Power splitting-based SWIPT with decode-and-forward full-duplex relaying," IEEE Transactions on Wireless Communications, vol. 15, no. 11, pp. 7561-7577, 2016.

[20] T. J. Tayag, M. B. Steer, J. F. Harvey, A. B. Yakovlev, and J. Davis, "Spatial power splitting and combining based on the Talbot effect," IEEE Microwave and Wireless Components Letters, vol. 12, no. 1, pp. 9-11, 2002.

[21] B. Clerckx, "Waveform optimization for SWIPT with nonlinear energy harvester modeling," in 20th International ITG Workshop on Smart Antennas (WSA 2016), pp. 1-5, Munich, Germany, March 2016.

[22] K.-H. Liu, "Outage-optimal relay selection for energyharvesting relays based on power splitting," in 2015 International Conference on Wireless Communications \& Signal Processing (WCSP), pp. 1-6, Nanjing, China, October 2015.

[23] K. Xiong, B. Wang, and K. R. Liu, "Rate-energy region of SWIPT for MIMO broadcasting under nonlinear energy harvesting model," IEEE Transactions on Wireless Communications, vol. 16, no. 8, pp. 5147-5161, 2017.

[24] K.-L. Wong, Compact and Broadband Microstrip Antennas, John Wiley \& Sons, Hoboken, NJ, USA, 2004. 


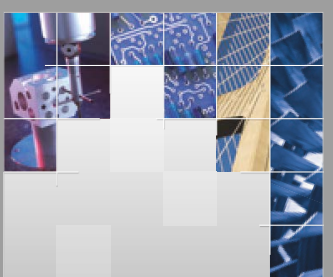

\section{Enfincering}
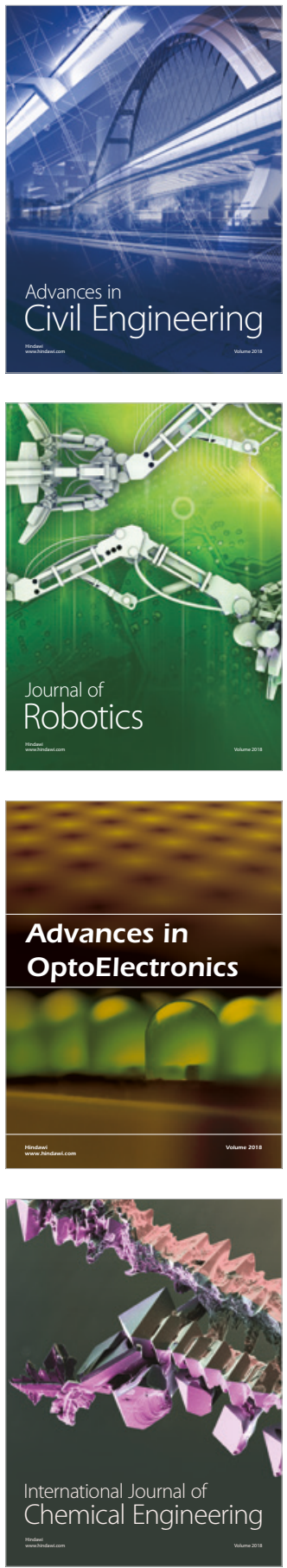

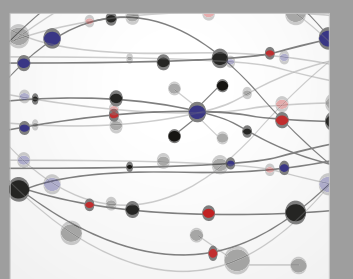

\section{Rotating \\ Machinery}

The Scientific World Journal

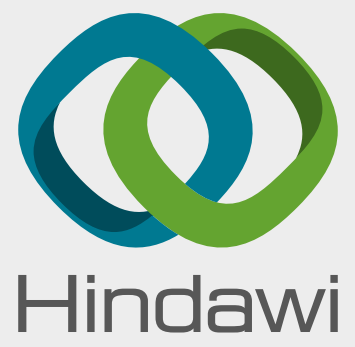

Submit your manuscripts at

www.hindawi.com
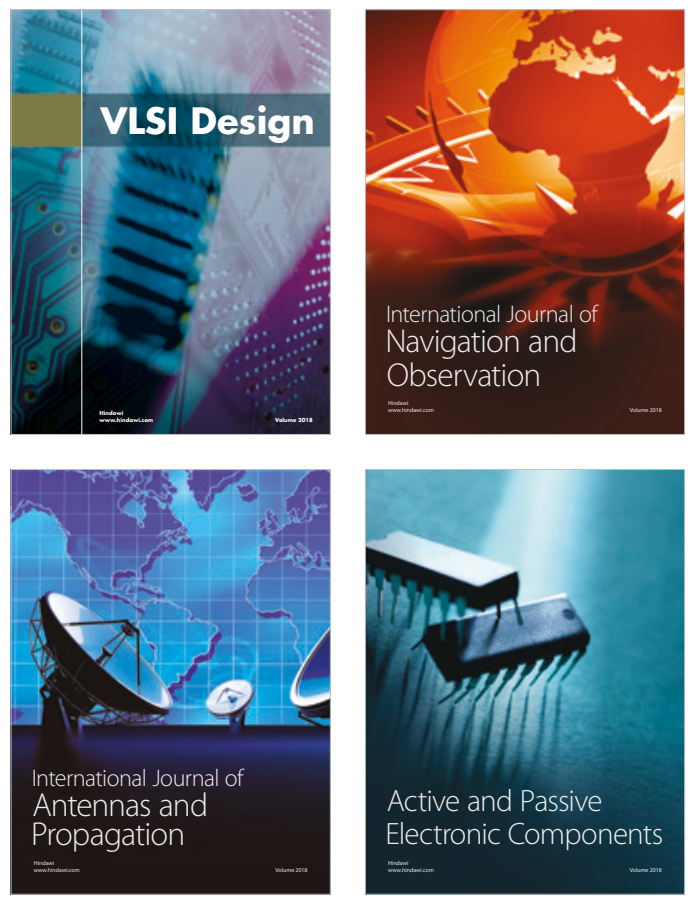
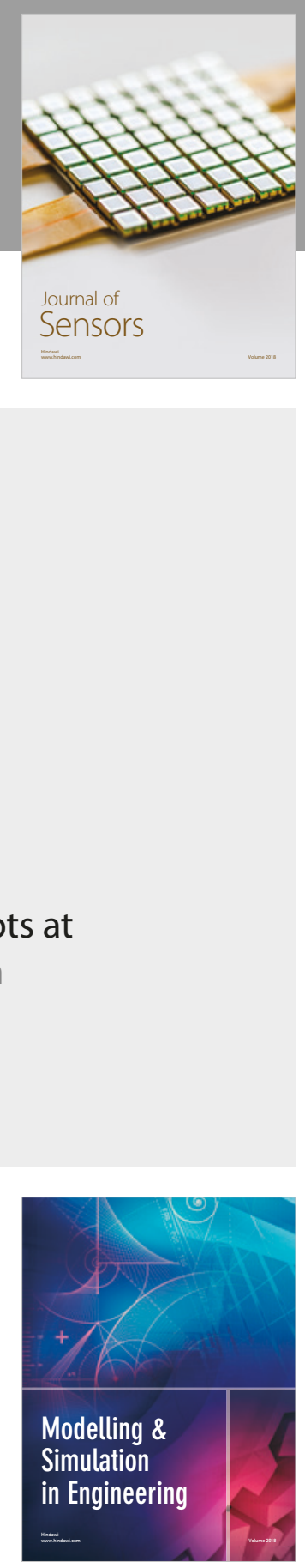

\section{Advances \\ Multimedia}
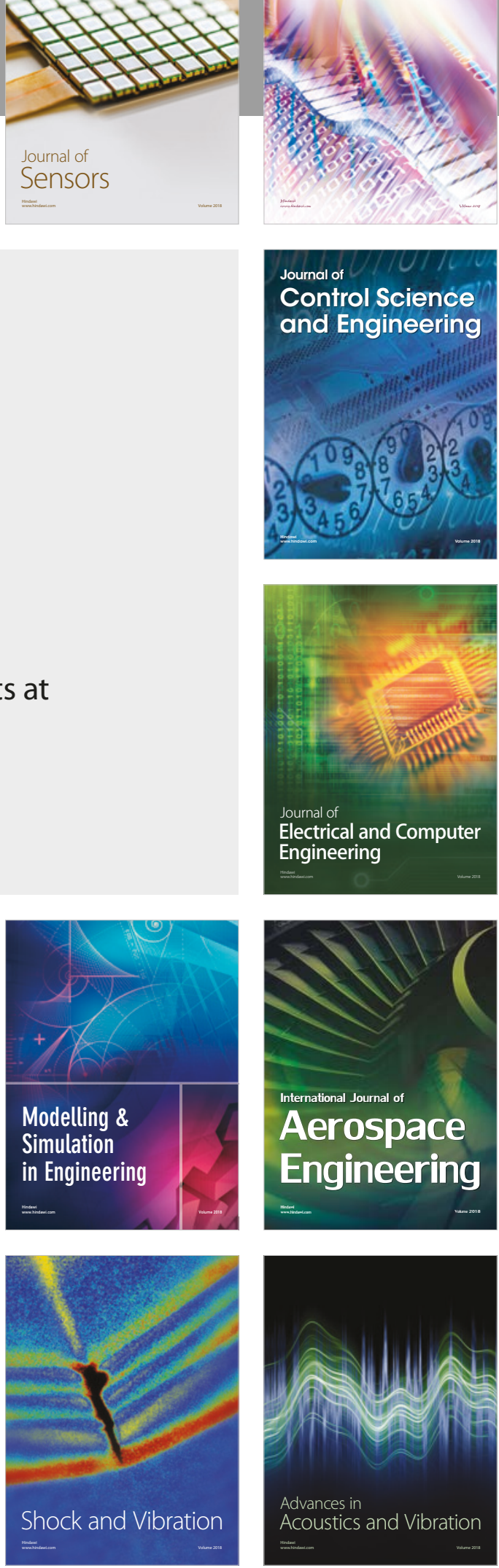